\title{
A Short Proof of the Hajnal-Szemerédi Theorem on Equitable Colouring
}

\author{
H. A. KIERSTEAD ${ }^{1 \dagger}$ and A. V. KOSTOCHKA ${ }^{2 \ddagger}$ \\ ${ }^{1}$ Department of Mathematics and Statistics, Arizona State University, Tempe, AZ 85287, USA \\ (e-mail: kierstead@asu.edu) \\ ${ }^{2}$ Department of Mathematics, University of Illinois, Urbana, IL 61801, USA \\ and \\ Institute of Mathematics, Novosibirsk, 630090, Russia \\ (e-mail: kostochk@math.uiuc.edu)
}

Received 7 August 2006; revised 28 March 2007

\begin{abstract}
A proper vertex colouring of a graph is equitable if the sizes of colour classes differ by at most one. We present a new shorter proof of the celebrated Hajnal-Szemerédi theorem: for every positive integer $r$, every graph with maximum degree at most $r$ has an equitable colouring with $r+1$ colours. The proof yields a polynomial time algorithm for such colourings.
\end{abstract}

\section{Introduction}

An equitable k-colouring of a graph $G$ is a proper $k$-colouring, for which any two colour classes differ in size by at most one. Equitable colourings naturally arise in some scheduling, partitioning, and load-balancing problems [1, 15, 16]. Pemmaraju [13] and Janson and Ruciński [6] used equitable colourings to derive deviation bounds for sums of random variables that exhibit limited dependence. In 1964 Erdős [3] conjectured that any graph with maximum degree $\Delta(G) \leqslant r$ has an equitable $(r+1)$-colouring. This conjecture was proved in 1970 by Hajnal and Szemerédi [5] with a surprisingly long and complicated argument. Recently, Mydlarz and Szemerédi [11] found a polynomial time algorithm for such colourings.

In search of an easier proof, Seymour [14] strengthened Erdős's conjecture by asking whether every graph with minimum degree $\delta(G) \geqslant \frac{k}{k+1}|G|$ contains the $k$ th power of a Hamiltonian cycle. (If $|G|=(r+1)(s+1)$ and $\Delta(G) \leqslant r$ then $\delta(\bar{G}) \geqslant \frac{s}{s+1}|G|$; each $(s+1)$-interval of an sth power of a Hamiltonian cycle in $\bar{G}$ is an independent set in $G$.) The case $k=1$ is Dirac's theorem and the case $k=2$ is Pósa's conjecture. Fan and Kierstead [4] proved Pósa's conjecture with 'cycle'

\footnotetext{
$\dagger$ Research of this author is supported in part by NSA grant MDA 904-03-1-0007.

$\ddagger$ Research of this author is supported in part by NSF grants DMS-0400498 and DMS-0650784, and by grant 05-0100816 of the Russian Foundation for Basic Research.
} 
replaced by 'path'. Komlós, Sárkőzy and Szemerédi [7] proved Seymour's conjecture for graphs with sufficiently many (in terms of $k$ ) vertices. Neither of these partial results has a simple proof. In fact, Komlós, Sárkőzy and Szemerédi [7] use the Regularity Lemma, the Blow-up Lemma and the Hajnal-Szemerédi theorem.

A different strengthening was suggested recently by Kostochka and Yu $[9,10]$. In the spirit of Ore's theorem on Hamiltonian cycles [12], they conjectured that every graph in which $d(x)+$ $d(y) \leqslant 2 r$ for every edge $x y$ has an equitable $(r+1)$-colouring.

In this paper we present a short proof of the Hajnal-Szemerédi theorem and present another polynomial time algorithm that constructs an equitable $(r+1)$-colouring of any graph $G$ with maximum degree $\Delta(G) \leqslant r$. Our approach is similar to the original method, but a discharging argument leads to a simpler conclusion. Our techniques have paid further dividends. In another paper we will prove the above conjecture of Kostochka and Yu $[9,10]$ in a stronger form: with $2 r+1$ in place of $2 r$. They also yield partial results towards the Chen-Lih-Wu conjecture [2] about equitable $r$-colourings of $r$-regular graphs and towards a list analogue of Hajnal-Szemerédi theorem (see [8] for definitions).

Most of our notation is standard; possible exceptions include the following. For a vertex $y$ and set of vertices $X, N_{X}(y):=N(y) \cap X$ and $d_{X}(y):=\left|N_{X}(y)\right|$. If $\mu$ is a function on the edge set, then $\mu(A, B):=\sum_{x y \in E(A, B)} \mu(x, y)$, where $E(A, B)$ is the set of edges joining a vertex in $A$ to a vertex in $B$. For a function $f: V \rightarrow Z$, the restriction of $f$ to $W \subseteq V$ is denoted by $f \mid W$. Functions are viewed formally as sets of ordered pairs. Thus, if $u \notin V$ then $g:=f \cup\{(u, \gamma)\}$ is the extension of $f$ to $V \cup\{u\}$ such that $g(u)=\gamma$.

\section{Main result}

Let $G$ be a graph with $s(r+1)$ vertices satisfying $\Delta(G) \leqslant r$. A nearly equitable $(r+1)$-colouring of $G$ is a proper colouring $f$, whose colour classes all have size $s$ except for one small class $V^{-}=V^{-}(f)$ with size $s-1$ and one large class $V^{+}=V^{+}(f)$ with size $s+1$. Given such a colouring $f$, define an auxiliary digraph $H=H(G, f)$ as follows. The vertices of $H$ are the colour classes of $f$, and a directed edge $V W$ belongs to $E(H)$ if and only if some vertex $y \in V$ has no neighbours in $W$. In this case we say that $y$ is movable to $W$. Call $W \in V(H)$ accessible if $V^{-}$is reachable from $W$ in $H$. Note that $V^{-}$is trivially accessible. Let $\mathscr{A}=\mathscr{A}(f)$ denote the family of accessible classes, $A:=\bigcup \mathscr{A}$ and $B:=V(G) \backslash A$. Let $m:=|\mathscr{A}|-1$ and $q:=r-m$. Then $|A|=(m+1) s-1$ and $|B|=(r-m) s+1$. Let $y \in B$. Since $y$ cannot be moved to $A$,

$$
d_{A}(y) \geqslant m+1 \text { and hence } d_{B}(y) \leqslant q-1 \text {. }
$$

Lemma 2.1. If $G$ has a nearly equitable $(r+1)$-colouring $f$ whose large class $V^{+}$is accessible, then $G$ has an equitable $(r+1)$-colouring.

Proof. Let $\mathscr{P}:=V_{1} \ldots V_{k}$ be a path in $H(G, f)$ from $V_{1}:=V^{+}$to $V_{k}:=V^{-}$. This means that for each $j=1, \ldots, k-1, V_{j}$ contains a vertex $y_{j}$ that has no neighbours in $V_{j+1}$. So, if we move $y_{j}$ to $V_{j+1}$ for $j=1, \ldots, k-1$, then we obtain an equitable $(r+1)$-colouring of $G$.

Suppose $V^{+} \subseteq B$. If $A=V^{-}$then $|E(A, B)| \leqslant r\left|V^{-}\right|=r(s-1)<1+r s=|B|$, a contradiction to (2.1). Thus $m+1=|\mathscr{A}| \geqslant 2$. Call a class $V \in \mathscr{A}$ terminal if $V^{-}$is reachable from every 
class $W \in \mathscr{A} \backslash\{V\}$ in the digraph $H-V$. Trivially, $V^{-}$is non-terminal. Every non-terminal class $W$ partitions $\mathscr{A} \backslash\{W\}$ into two parts $\mathscr{S}_{W}$ and $\mathscr{T}_{W} \neq \emptyset$, where $\mathscr{S}_{W}$ is the set of classes that can reach $V^{-}$in $H-W$. Choose a non-terminal class $U$ so that $\mathscr{A}^{\prime}:=\mathscr{T}_{U} \neq \emptyset$ is minimal. Then every class in $\mathscr{A}^{\prime}$ is terminal and no class in $\mathscr{A}^{\prime}$ has a vertex movable to any class in $\left(\mathscr{A} \backslash \mathscr{A}^{\prime}\right) \backslash\{U\}$. Set $t:=\left|\mathscr{A}^{\prime}\right|$ and $A^{\prime}:=\bigcup \mathscr{A}^{\prime}$. Thus every $x \in A^{\prime}$ satisfies

$$
d_{A}(x) \geqslant m-t .
$$

Call an edge $z y$ with $z \in W \in \mathscr{A}^{\prime}$ and $y \in B$ a solo edge if $N_{W}(y)=\{z\}$. The ends of the solo edges are called solo vertices and the vertices joined by the solo edges are called special neighbours of each other. Let $S_{z}$ denote the set of special neighbours of $z$ and let $S^{y}$ denote the set of special neighbours of $y$ in $A^{\prime}$. Let $y \in B$. Since at most $r-\left(m+1+d_{B}(y)\right)$ colour classes in $A$ have more than one neighbour of $y$,

$$
\left|S^{y}\right| \geqslant t-q+1+d_{B}(y) .
$$

Lemma 2.2. If there exists $W \in \mathscr{A}^{\prime}$ such that no solo vertex in $W$ is movable to a class in $\mathscr{A} \backslash\{W\}$, then $q+1 \leqslant t$. Furthermore, every vertex $y \in B$ is solo.

Proof. Let $S$ be the set of solo vertices in $W$ and $D:=W \backslash S$. Then every vertex in $N_{B}(S)$ has at least one neighbour in $W$ and every vertex in $B \backslash N_{B}(S)$ has at least two neighbours in $W$. It follows that $\left.|E(W, B)| \geqslant\left|N_{B}(S)\right|+2\left(|B|-\mid N_{B}(S)\right) \mid\right)$. Since no vertex in $S$ is movable, every $z \in S$ satisfies $d_{B}(z) \leqslant q$. By (2.2), every vertex $x \in W$ satisfies $d_{B}(x) \leqslant t+q$. Thus, using $s=|W|=|S|+|D|$,

$$
q s+q|D|+2=2(q s+1)-q|S| \leqslant|E(W, B)| \leqslant q|S|+(t+q)|D| \leqslant q s+t|D| .
$$

It follows that $q+1 \leqslant t$. Moreover, by (2.3) every $y \in B$ satisfies $\left|S^{y}\right| \geqslant t-q+d_{B}(y) \geqslant 1$. Thus $y$ is solo.

Lemma 2.3. There exists a solo vertex $z \in W \in \mathscr{A}^{\prime}$ such that either $z$ is movable to a class in $\mathscr{A} \backslash\{W\}$ or z has two nonadjacent special neighbours in $B$.

Proof. Suppose not. Then by Lemma 2.2 every vertex in $B$ is solo. Moreover, $S_{z}$ is a clique for every solo vertex $z \in A^{\prime}$. Consider a weight function $\mu$ on $E\left(A^{\prime}, B\right)$ defined by

$$
\mu(x y):= \begin{cases}\frac{q}{\left|S_{x}\right|} & \text { if } x y \text { is solo, } \\ 0 & \text { if } x y \text { is not solo. }\end{cases}
$$

For $z \in A^{\prime}$ we have $\mu(z, B)=\left|S_{z}\right| \frac{q}{\left|S_{z}\right|}=q$ if $z$ is solo; otherwise $\mu(z, B)=0$. Thus $\mu\left(A^{\prime}, B\right) \leqslant$ $q\left|A^{\prime}\right|=q$ st. On the other hand, consider $y \in B$. Let $c_{y}:=\max \left\{\left|S_{z}\right|: z \in S^{y}\right\}$, say $c_{y}=\left|S_{z}\right|, z \in$ $S^{y}$. Using that $S_{z}$ is a clique and $(2.1), c_{y}-1 \leqslant d_{B}(y) \leqslant q-1$. So $c_{y} \leqslant q$. Together with (2.3) this yields

$$
\mu\left(A^{\prime}, y\right)=\sum_{z \in S^{y}} \frac{q}{\left|S_{z}\right|} \geqslant\left|S^{y}\right| \frac{q}{c_{y}} \geqslant\left(t-q+c_{y}\right) \frac{q}{c_{y}}=(t-q) \frac{q}{c_{y}}+q \geqslant t .
$$

Thus $\mu\left(A^{\prime}, B\right) \geqslant t|B|=t(q s+1)>q s t \geqslant \mu\left(A^{\prime}, B\right)$, a contradiction. 
We are now ready to prove the Hajnal-Szemerédi theorem.

Theorem 2.4. If $G$ is a graph satisfying $\Delta(G) \leqslant r$, then $G$ has an equitable $(r+1)$-colouring.

Proof. We may assume that $|G|$ is divisible by $r+1$. To see this, suppose that $|G|=s(r+1)-$ $p$, where $p \in[r]$. Let $G^{\prime}:=G+K^{p}$. Then $\left|G^{\prime}\right|$ is divisible by $r+1$ and $\Delta\left(G^{\prime}\right) \leqslant r$. Moreover, the restriction of any equitable $(r+1)$-colouring of $G^{\prime}$ to $G$ is an equitable $(r+1)$-colouring of $G$.

Argue by induction on $\|G\|$. The base step $\|G\|=0$ is trivial, so consider the induction step $\|G\| \geqslant 1$. Let $e=x y$ be an edge of $G$. By the induction hypothesis there exists an equitable $(r+1)$-colouring $f_{0}$ of $G-e$. Hence we are done unless some colour class $V$ contains both $x$ and $y$. Since $d(x) \leqslant r$, there exists another class $W$ such that $x$ is movable to $W$. Doing so yields a nearly equitable $(r+1)$-colouring $f$ of $G$ with $V^{-}(f)=V \backslash\{x\}$ and $V^{+}(f)=W \cup\{x\}$. We now show by a secondary induction on $q(f)$ that $G$ has an equitable $(r+1)$-colouring.

If $V^{+} \in \mathscr{A}$ then we are done by Lemma 2.1; in particular, the base step $q=0$ holds. Otherwise, by Lemma 2.3 there exists a class $W \in \mathscr{A}^{\prime}$, a solo vertex $z \in W$ and a vertex $y_{1} \in S_{z}$ such that either $z$ is movable to a class $X \in \mathscr{A} \backslash\{W\}$ or $z$ is not movable in $\mathscr{A}$ and there exists another vertex $y_{2} \in S_{z}$, which is not adjacent to $y_{1}$. By (2.1) and the primary induction hypothesis, there exists an equitable $q$-colouring $g$ of $B^{-}:=B \backslash\left\{y_{1}\right\}$. Let $A^{+}:=A \cup\left\{y_{1}\right\}$.

Case 1: $z$ is movable to $X \in \mathscr{A}$. Move $z$ to $X$ and $y_{1}$ to $W \backslash\{z\}$ to obtain a nearly equitable $(m+1)$-colouring $\varphi$ of $A^{+}$. Since $W \in \mathscr{A}^{\prime}(f), V^{+}(\varphi)=X \cup\{z\} \in \mathscr{A}(\varphi)$. By Lemma 2.1, $A^{+}$ has an equitable $(m+1)$-colouring $\varphi^{\prime}$. Then $\varphi^{\prime} \cup g$ is an equitable $(r+1)$-colouring of $G$.

Case 2: $z$ is not movable to any class in $\mathscr{A}$. Then $d_{A^{+}}(z) \geqslant d_{A}(z)+1 \geqslant m+1$. Thus $d_{B^{-}}(z) \leqslant$ $q-1$. So we can move $z$ to a colour class $Y \subseteq B$ of $g$ to obtain a new colouring $g^{\prime}$ of $B^{*}:=$ $B^{-} \cup\{z\}$. Also, move $y_{1}$ to $W$ to obtain an $(m+1)$-colouring $\psi$ of $A^{*}:=V(G) \backslash B^{*}$. Set $\psi^{\prime}:=$ $\psi \cup g^{\prime}$. Then $\psi^{\prime}$ is a nearly equitable colouring of $G$ with $A^{*} \subseteq A\left(\psi^{\prime}\right)$. Moreover, $y_{2}$ is movable to $W^{*}:=W \cup\left\{y_{1}\right\} \backslash\{z\}$. Thus $q\left(\psi^{\prime}\right)<q(f)$, and so by the secondary induction hypothesis, $G$ has an equitable $(r+1)$-colouring $\psi^{\prime \prime}$.

\section{A polynomial algorithm}

Our proof clearly yields an algorithm. However it may not be immediately clear that its running time is polynomial. The problem lies in the secondary induction, where we may apply Case 2 $O(r)$ times, each time calling the algorithm recursively. Lemma 2.2 is crucial here; it allows us to claim that when we are in Case 2 (doing much work) we make much progress. As above, $G$ is a graph satisfying $\Delta(G) \leqslant r$ and $|G|=s(r+1)=: n$. Let $f$ be a nearly equitable $(r+1)$-colouring of $G$.

Theorem 3.1. There exists an algorithm $\mathscr{P}^{\prime}$ that from input $(G, f)$ constructs an equitable $(r+$ 1)-colouring of $G$ in $c(q+1) n^{3}$ steps.

Proof. We shall show that the construction in the proof of Theorem 2.4 can be accomplished in the stated number of steps. Argue by induction on $q$. The base step $q=0$ follows immediately from Lemma 2.1 and the observation that the construction of $H$ and the recolouring can be carried 
out in $\frac{1}{4} c n^{3}$ steps. Now consider the induction step. In $\frac{1}{4} c n^{3}$ steps construct $\mathscr{A}, \mathscr{A}^{\prime}, B, W, z, y_{1}$. Using the induction hypothesis on the input $\left(G\left[B^{-}\right], f \mid B^{-}\right)$, construct the colouring $g$ of $B^{-}$in $c\left(q\left(f \mid B^{-}\right)+1\right)(q s)^{3} \leqslant c q n^{3}$ steps. In $\frac{1}{4} c n^{3}$ steps determine whether Case 1 or Case 2 holds.

If Case 1 holds, construct the recolouring $\varphi^{\prime}$ in $\frac{1}{4} c n^{3}$ steps. This yields an equitable $(r+1)$ colouring $g \cup \varphi^{\prime}$ in a total of $\frac{3}{4} c n^{3}+c q n^{3} \leqslant c(q+1) n^{3}$.

If Case 2 holds then, by Lemma $2.2, q+1 \leqslant t$. Thus we used only $\frac{1}{8} c q n^{3}$ steps to construct $g$. Use an additional $\frac{1}{4} c n^{3}$ steps to extend $g$ to $\psi^{\prime}$. Notice that $W^{*}$ is non-terminal in $\psi^{\prime}$. Thus we can choose $\mathscr{A}^{\prime}\left(\psi^{\prime}\right)$ so that $A^{\prime}\left(\psi^{\prime}\right) \subseteq B$. If Case 1 holds for $\psi^{\prime}$ then, as above, we can construct an equitable colouring in an additional $\frac{1}{4} c n^{3}+\frac{1}{8} c q n^{3}$ steps. So the total number of steps is at most $c(q+1) n^{3}$. Otherwise, by Lemma 2.2, $q\left(\psi^{\prime}\right)<\frac{1}{2} q$. Thus, by the induction hypothesis, we can finish the construction in $c \frac{q n^{3}}{16}$ additional steps. Therefore, the total number of steps is less than $c(q+1) n^{3}$.

Theorem 3.2. There is an algorithm $\mathscr{P}$ of complexity $O\left(n^{5}\right)$ that constructs an equitable $(r+$ $1)$-colouring of any graph $G$ satisfying $\Delta(G) \leqslant r$ and $|G|=n$.

Proof. As above, we may assume that $n$ is divisible by $r+1$. Let $V(G):=\left\{v_{1}, \ldots, v_{n}\right\}$. Delete all the edges from $G$ to form $G_{0}$ and let $f_{0}$ be an equitable colouring of $G_{0}$. Now, for $i=1, \ldots, n-1$, do the following.

(i) Add back all the edges of $G$ incident with $v_{i}$ to form $G_{i}$.

(ii) If $v_{i}$ has no neighbours in its colour class in $f_{i-1}$, then set $f_{i}:=f_{i-1}$.

(iii) Otherwise, move $v_{i}$ to a colour class that has no neighbours of $v_{i}$ to form a nearly equitable colouring $f_{i-1}^{\prime}$ of $G_{i}$. Then apply $\mathscr{P}^{\prime}$ to $\left(G_{i}, f_{i-1}^{\prime}\right)$ to get an equitable $(r+1)$-colouring $f_{i}$ of $G_{i}$.

Then $f_{n-1}$ is an equitable $(r+1)$-colouring of $G_{n-1}=G$. Since we have only $n-1$ stages and each stage runs in $O\left(n^{4}\right)$ steps, the total complexity is $O\left(n^{5}\right)$.

\section{Acknowledgement}

We would like to thank J. Schmerl for many useful comments.

\section{References}

[1] Blazewicz, J., Ecker, K., Pesch, E., Schmidt, G. and Weglarz, J. (2001) Scheduling Computer and Manufacturing Processes, 2nd edn, Springer.

[2] Chen, B.-L., Lih, K.-W. and Wu, P.-L. (1994) Equitable coloring and the maximum degree. Europ. J. Combin. 15 443-447.

[3] Erdős, P. (1964) Problem 9. In Theory of Graphs and its Applications (M. Fiedler, ed.), Czech. Academy of Sciences, Prague, p. 159.

[4] Fan, G. and Kierstead, H. A. (1996) Hamiltonian square paths. J. Combin. Theory Ser. B 67 167-182.

[5] Hajnal, A. and Szemerédi, E. (1970) Proof of a conjecture of P. Erdős. In Combinatorial Theory and its Application (P. Erdős, A. Rényi, and V. T. Sós, eds), North-Holland, London, pp. 601-623.

[6] Janson, S. and Ruciński, A. (2002) The infamous upper tail. Random Struct. Alg. 20 317-342.

[7] Komlós, J., Sárkôzy, G. and Szemerédi, E. (1998) Proof of the Seymour conjecture for large graphs. Ann. Combin. 1 43-60. 
[8] Kostochka, A. V., Pelsmajer, M. J. and West, D. B. (2003) A list analogue of equitable coloring. J. Graph Theory 44 166-177.

[9] Kostochka, A. V. and Yu, G. (2006) Extremal problems on packing of graphs. Oberwolfach Reports 1 $55-57$.

[10] Kostochka, A. V. and Yu, G. (2007) Ore-type graph packing problems. Combin. Probab. Comput. 16 $167-169$.

[11] Mydlarz, M. and Szemerédi, E. Algorithmic Brooks' theorem. Manuscript.

[12] Ore, O. (1960) Note on Hamilton circuits. Amer. Math. Monthly 6755.

[13] Pemmaraju, S. V. (2001) Equitable colorings extend Chernoff-Hoeffding bounds. In Proc. 5th International Workshop on Randomization and Approximation Techniques in Computer Science (APPROX-RANDOM 2001), pp. 285-296.

[14] Seymour, P. (1974) Problem section. In Combinatorics: Proc. British Combinatorial Conference 1973 (T. P. McDonough and V. C. Mavron, eds), Cambridge University Press, pp. 201-202.

[15] Smith, B. F., Bjorstad, P. E. and Gropp, W. D. (1996) Domain Decomposition: Parallel Multilevel Methods for Elliptic Partial Differential Equations, Cambridge University Press.

[16] Tucker, A. (1973) Perfect graphs and an application to optimizing municipal services. SIAM Review 15 585-590. 